\title{
TTR
}

Traduction, terminologie, rédaction

\section{Anthony Pym, Miriam Shlesinger, Daniel Simeoni, dirs. Beyond Descriptive Translation Studies. Investigation in homage to Gideon Toury. Amsterdam et Philadelphia, Benjamins, 2008, i-xii, 417 p.}

\section{Aurelia Klimkiewicz}

Volume 22, numéro 2, 2e semestre 2009

Littérature comparée et traductologie littéraire : convergences et divergences

Comparative Literature and Literary Translation Studies: Points of Convergence and Divergence

URI : https://id.erudit.org/iderudit/044835ar

DOI : https://doi.org/10.7202/044835ar

Aller au sommaire du numéro

Éditeur(s)

Association canadienne de traductologie

ISSN

0835-8443 (imprimé)

1708-2188 (numérique)

Découvrir la revue

Citer ce compte rendu

Klimkiewicz, A. (2009). Compte rendu de [Anthony Pym, Miriam Shlesinger, Daniel Simeoni, dirs. Beyond Descriptive Translation Studies. Investigation in homage to Gideon Toury. Amsterdam et Philadelphia, Benjamins, 2008, i-xii, 417 p.] TTR, 22(2), 255-264. https://doi.org/10.7202/044835ar

Tous droits réservés @ TTR: traduction, terminologie, rédaction — Les auteurs, 2010
Ce document est protégé par la loi sur le droit d'auteur. L’utilisation des services d'Érudit (y compris la reproduction) est assujettie à sa politique d'utilisation que vous pouvez consulter en ligne.

https://apropos.erudit.org/fr/usagers/politique-dutilisation/ 
and consideration of what exactly "ideology" is, and would have benefited from more theoretical discussion of this key term. Instead, "feminist translation" is used here as a foil, representing the extreme of ideology in translation-as if all texts are not ideological, whether or not this is consciously expressed and assumed by their authors.

Overall, while the clarity of expression and thoroughness of Leonardi's approach is admirable, and I certainly benefited from confronting such an unequivocal exposition of the ideals and methods of equivalence-based approaches to translation, as a (feminist) translator committed to identifying a text's ideology and engaging in it in terms of my own ideologies, I can only conclude that the question of differences in translation may not lie so much in gender as in the ideologies of translation theories.

\title{
Carolyn Shread Mount Holyoke College
}

\begin{abstract}
Anthony Pym, Miriam Shlesinger, Daniel Simeoni, dirs. Beyond Descriptive Translation Studies. Investigation in homage to Gideon Toury. Amsterdam et Philadelphia, Benjamins, 2008, i-xii, 417 p.
\end{abstract}

\section{Toury et le tournant descriptiviste}

Dans ce volume, il est question de réfléchir sur l'au-delà du descriptivisme élaboré dans les années soixante-dix par EvenZohar, Gideon Toury et José Lambert, au moment où la traductologie cherchait à s'affranchir des modèles théoriques plutôt statiques et normatifs issus de la linguistique moderne. Influencé par Nida, Catford et Even-Zohar, le travail de Toury est également indissociable des influences européennes, notamment du formalisme russe (Jakobson, Tynjanov) et de la sémiotique en France (Barthes, Genette, Greimas, Todorov) (Simeoni, p. 330). Ainsi, le descriptivisme introduit une transition vers le modèle orienté sur les fonctions sociales que la traduction assume dans le contexte cible. Cette approche, qui tient compte de la composante sociale, coïncide cependant avec les théories 
de la réception de Iser (1974) et de Jauss (1977) de l'École de Constance, qui, en abandonnant le texte lui-même comme objet d'études, se penchent sur la réception et les compétences $\mathrm{du}$ lecteur. Toutefois, cette transition méthodologique vers la réception est en quelque sorte portée par le post-structuralisme dans la mesure où elle annonce à la fois la mort de l'auteur et l'avènement du lecteur. Par conséquent, une série de déplacements permettent la réorganisation des ancrages théoriques et méthodologiques : de l'auteur au récepteur; de la genèse à la réception; de l'origine à la cible; de l'hypothèse à la vérification; de la spéculation à la description (objectivité); du texte source au texte cible. Le descriptivisme élargit ainsi la réflexion au-delà du linguistique et du textuel, tout en ramenant la traduction à son environnement social le plus immédiat. À la manière d'une pratique du soupçon qui rend explicites les dispositifs sociaux et les enjeux politiques et économiques dans lesquels s'inscrit l'activité traductive, la démarche de Toury dévoile les normes, les lois et les contraintes en tant que forces régulatrices de l'activité de traduction à laquelle participent des actants aux compétences spécifiques qui prennent des décisions concrètes quant à la sélection du texte à traduire, sa mise en circulation et sa réception ultérieure dans la société réceptrice.

En même temps, le descriptivisme a un impact direct sur la discipline, puisqu'il consolide le statut épistémologique des études traductologiques et légitime leur ambition scientifique. Pour le reste, il résistera avec aisance à la crise des représentations qui sévit dans les années 80 , au moment du virage culturaliste, alors que l'espace social se fragmente et le besoin pour les démarches critiques se fait sentir, démarches qui seraient plus subjectives, plus engagées et ouvertes à l'inclusion de différents types de voix et de perspectives. C'est d'ailleurs l'époque où l'on se tourne vers l'herméneutique pour expliquer les rouages complexes du processus interprétatif menant à la saisie du sens. De même, les années 90, marquées par les questions identitaires, laisseront intacte la théorie descriptive aux prises avec le raffinement méthodologique qui tente, entre autres, de dépasser l'étape descriptive en somme préliminaire (collecte et analyse de données) pour en dégager les hypothèses explicatives (interprétation des données) (Chesterman, p. 363), celles-ci étant 
indispensables à la compréhension subjective des phénomènes étudiés et à la contribution au savoir. En termes plus simples, en 1995, lors de la publication de son deuxième ouvrage Descriptive Translation Studies and Beyond, Toury abandonne la théorisation pour le questionnement (Simeoni, p. 331).

Mais les questions de pouvoir qui sont graduellement prises en charge par les traductologues, notamment par Lawrence Venuti, tout en révélant les pratiques sociales institutionnelles, débouchent sur une approche ethnographique qui, au lieu de scruter l'objet, met la démarche du chercheur au centre de l'analyse. À cet endroit, l'apport de Pierre Bourdieu - transplanté en traductologie par Daniel Simeoni et Jean-Marc Gouanvic (Merkle, p. 175) - consiste justement à amener l'agent social à l'auto-analyse et à la conscience de soi. D'un autre côté, le descriptivisme provoque par ricochet un regain d'intérêt pour l'histoire de la traduction et, par conséquent, participe indirectement au tournant post-colonialiste. Il ouvre en effet des pistes de réflexion étrangement en contradiction avec l'image traditionnelle qui veut que le traducteur soit un être désincarné, dépourvu de volonté et de substance, en soulignant au contraire le rôle actif et souvent pernicieux que jouait la traduction dans les tournants historiques importants et particulièrement dans le projet de colonisation.

Sur le plan méthodologique, le modèle de Toury est vertical, comme le souligne Denise Merkle : la norme vient toujours d'en haut et impose la soumission, ou encore, dans le cas d'une désobéissance, la sanction. Une telle perspective bloque la circulation horizontale entre les traducteurs eux-mêmes (Merkle, p. 179), par exemple, ou entre différents groupes linguistiques dans un espace social multiethnique. Par contre, Alexis Nouss remarque que la démarche descriptiviste est à la fois rigide et souple, puisqu'à côté des normes et des lois traductionnelles se trouvent des pratiques traductives transgressives comme la pseudo-traduction, la traduction indirecte et l'interférence (Nouss, p. 382). C'est surtout son caractère synchronique qui constitue une faiblesse méthodologique, parce que, en évacuant la dimension historique, Toury abolit du même coup le caractère relatif et non absolu de la norme comme phénomène historiquement situé et 
porteur d'une pensée de l'histoire (ibid., p. 383). Dans le même geste disparaît également l'histoire des contacts entre les langues, les cultures et les littératures qui se jouent aux frontières, dans un entre-deux, et non pas exclusivement à l'intérieur de l'espace national. Le noyau du problème de la théorie descriptive - ce qui ressort de la majorité des contributions du présent recueil - serait donc son enracinement dans le temps et dans l'espace puisque intimement liée à l'avènement de l'État, à sa naissance et à sa survie (Simeoni, p. 335).

\section{Après Toury}

De nos jours, si l'approche sociocognitive l'emporte sur le descriptivisme initial parce qu'elle s'occupe de l'agent lui-même et non pas de ce que lui impose la société (Merkle, p. 179), c'est à la suite d'un double décentrement, introduit par Bourdieu, du système vers l'institution et de l'institution vers l'agent. Dans le domaine de la traduction, une telle perspective coïncide avec le discours qui valorise les traducteurs et tentent de leur accorder une plus grande visibilité sur les scènes locales et mondiales. Si dans les années 90, la norme devint synonyme d'équivalence, aujourd'hui c'est le cas de la négociation, concept introduit en traductologie par Anthony Pym, qui met l'accent non seulement sur l'interaction entre l'individuel et le social, mais aussi sur le rôle actif que le traducteur assume dans le réseau professionnel. D'un autre côté, l'intérêt croissant à l'égard de l'interprétation instaure lentement une certaine coopération intra-disciplinaire, comme le précise Ebru Diriker (p. 209), du simple fait que l'approche descriptive repose sur le produit, tandis que les études interprétatives analysent le processus et, par conséquent, sont centrées sur l'agent lui-même. Ce rapprochement intradisciplinaire fait ressentir davantage le besoin d'une approche critique, étant donné que l'interprétation implique des pratiques sociales complexes qui sont parfois difficiles à détecter, surtout dans le cas de l'interprétation communautaire (p. 212).

Si les sociétés ne sont ni homogènes ni monolingues, si les systèmes littéraires ne sont pas autosuffisants, si la langue est dialogique par essence, si la traduction multiplie ses modalités, comment parler de la norme? Comment l'aborder dans le contexte 
actuel hautement mobile et surchargé par les configurations spatiales de plus en plus diversifiées? Et comment systématiser les phénomènes de traduction alors que celle-ci symbolise le déplacement lui-même (Lieven D'hulst, p. 229)? Même l'Israël qui sert de terrain d'observation à Toury échappe aujourd'hui à une systématisation du point de vue linguistique, culturel et social. Il n'est pas surprenant que les quatre premières contributions du recueil (Nitsa Ben-Ari, Hannah Amit-Kochavi, Mahmoud Kayyal et Rachel Weissbrod) en témoignent en insistant sur le caractère hétérogène de la société israélienne et sur son histoire profondément inscrite dans l'exil et le déplacement. Et que dire de la diaspora? Sherry Simon soulève justement les tensions linguistiques des sujets diasporiques multilingues insatisfaits d'une seule langue (Simon, p. 68). Les pratiques de la traduction, de l'autotraduction, de la traduction indirecte ou de la traduction collaborative ne sont que des tentatives de pallier l'indécidabilité quant à la langue d'appartenance ou d'écriture. Encore une fois, le descriptivisme est impuissant à traiter ces questions du simple fait que le multilinguisme se dissout simplement dans la norme.

Deux pistes permettent cependant de sortir de l'impasse imposée par la norme : l'éducation et la politique culturelle. Selon Michael Cronin (p. 266), la traduction pourrait exercer un rôle éducationnel en créant l'appartenance globale à force d'anticiper le récepteur futur et de forger de nouvelles habitudes de réception inscrites dans un horizon lointain à atteindre. Contrairement à une perspective mondiale faisant fi du local, entre autres, par l'usage de l'anglais comme langue de communication transparente, cette nouvelle appartenance logerait dans le local et serait au service de la diversité, de l'interconnectivité tout en se portant à la défense de la traduction. Le tourisme, le marché immobilier et la migration sont des lieux d'émergence de cette nouvelle appartenance qui mettent de l'avant le concept de denizen [résident d'origine étrangère] et signalent le " shift from recognition to implication » (p. 270).

C'est Even-Zohar qui introduit la problématique de la planification de la culture qui maintient l'ordre au sein de la société (p. 278). On y entend à la fois Tynjanov et Lotman, qui ont contribué à une vision plutôt spatiale du système littéraire et 
culturel ayant pourtant la capacité d'introduire du nouveau et de réorganiser l'ensemble du système sans perturber sa cohérence ou l'unité culturelle. Une nouvelle planification de la culture exige de l'énergie parce qu'elle s'impose soit lors d'un tournant historique majeur (p. ex., guerre, révolution, etc.), soit par le biais d'une action sociale contre le statu quo. Lélément nouveau - le texte traduit, la valeur importée, la pression de la part des migrants dialogise ainsi le système, dialogisme étant synonyme de vitalité culturelle. Parfois, la société se mobilise pour traiter de ses questions, comme c'était le cas au Québec il y a quelques années autour de la place qu'il fallait accorder à la littérature migrante au sein du système littéraire local. Even-Zohar vise également les spécialistes de la traduction qui devraient peut-être s'investir à fond dans la planification de la culture, étant situés à la jonction entre le domestique et l'étranger, entre le local et le mondial.

\section{Au-delà de Toury}

Outre la norme, les concepts touriens le plus souvent abordés dans le présent volume sont les pseudo-traductions, les interférences et les textes ayant le statut de traduction (assumed translations). La liberté d'exploration et d'innovation n'est pas étrangère à la démarche de Toury qui, tout aussi bien que Bourdieu, est critique à l'égard du pouvoir uniformisateur de l'État et de ses institutions (Simeoni, p. 335). Reste toutefois à résoudre le problème épistémologique concernant la classification des concepts et des catégories hybrides alors que l'ontologie occidentale repose sur une logique binaire. À juste titre, Andrea Rizzi suggère d'étudier la Renaissance, durant laquelle la traduction n'était pas encore clairement délimitée comme activité autonome et se manifestait surtout sous forme de pseudo-traduction (p. 157). Ce détour historique, tout en relativisant la norme, pourrait aider à comprendre pourquoi les frontières entre traduction et écriture disparaissent dans l'auto-traduction et quel effet ce brouillage provoque sur la norme (D'hulst, p. 229).

Il ne relève toutefois pas du hasard que les concepts qui ont retenu l'attention des chercheurs échappent à la classification et la systématisation qu'impose habituellement la norme. Le multilinguisme, la migration, la mondialisation et autres types 
de déplacement introduisent le contexte de transition de la culture cible comme système culturel autonome vers les relations transnationales qui créent ensemble le système mondial (the world system), ce qui exige que le local soit étudié en fonction de la place que la culture occupe dans le système mondial, étant donné que certaines cultures sont plus proactives que d'autres. En Hollande, par exemple, on privilégie l'accommodation active (Heilbron, pp. 187-188). D’un autre côté, comme le précise Gisèle Sapiro, les contextes source et cible ne peuvent plus être étudiés en tant qu'entités homogènes, mais comme des entités qui présentent des logiques et des contraintes parfois contradictoires, en concurrence ou en conflit (p. 201). Il faudrait donc inscrire le descriptivisme dans le tournant international (Delabastita, p. 233), c'est-àdire proposer des approches plus nuancées qui mettraient en perspective le type du circuit de transfert, la position du texte dans le contexte source, la position des médiateurs en fonction des conditions d'acquisition de la compétence linguistique et en fonction du rapport à la culture cible (p. ex., le parcours migratoire privilégie la traduction sourcière), ainsi que l'habitus $\mathrm{du}$ traducteur en tant que rapport individuel au social (Sapiro, p. 205). S'ajoute à cela le rapport du traducteur au texte source en tant que différence, élément dont la démarche descriptive ne tient pas compte alors que la traduction est avant tout lecture laissant son empreinte dans le texte cible en fonction des stratégies privilégiées par le traducteur : la purification, le consentement ou la résistance (Merkle, pp. 178-179).

Il reste également à réfléchir sur le statut du traducteur comme agent. Encore une fois, il y a grand besoin de créer de catégories hybrides ou simplement de nouveaux concepts. Puisqu'il y a de plus en plus de cas de figure, n'est-il pas devenu nécessaire de concevoir chacun des cas par rapport aux autres qui font partie d'un ensemble? Salah Basalamah, par un détour archéologique, souligne en effet que même le concept d'auteur - si central dans l'économie capitaliste - n'est que le fruit d'un dispositif sociopolitique et économique bien précis. Dans la situation actuelle où le multilinguisme est de plus en plus présent, comme le précise Reine Meylaerts, il serait important de réfléchir sur un habitus transculturel pour réduire le décalage et la dissonance auxquels sont confrontés de nombreux sujets 
multilingues vivant dans la société d'accueil (p. 95). Dans la même logique, il est de plus en plus difficile de défendre le traducteur comme agent qui serait entièrement en harmonie avec le « nous » collectif, puisqu'il est de plus en plus exposé aux enjeux interculturels et impliqué dans les réseaux transnationaux.

Bien que la méthode descriptive ait donné fruit à de nombreuses recherches encadrées par une démarche scientifique rigoureuse ayant un objectif heuristique, le sujet traduisant et l'éthique sont deux grands oubliés du descriptivisme. D'un côté, il s'agirait d'un simple changement paradigmatique; de l'autre, il faudrait changer la perception, voire la philosophie de la traduction. En accord avec le courant postmoderne qui permet de penser l'éclatement, la fragmentation et le relativisme et en mettant l'accent sur le choix individuel sur lequel repose la posture éthique, la réflexion en traduction aura à gagner en se tournant vers le traducteur lui-même. La théorie de la lecture semble toutefois insuffisante; ce qu'il faut, c'est une herméneutique du sujet traduisant qui permettrait d'explorer comment ce sujet qui traduit inscrit l'expérience du monde et le savoir dans sa carte mentale. Où se loge l'activité traductive et comment réorganiset-elle le système déjà en place? Quel type de récit abrite les connaissances? L'idée n'est pas neuve : elle fait partie du projet critique qu'Antoine Berman écrivait à la hâte sur son lit de mort. Dans les "Tâches à la traductologie ", il a d'ailleurs formulé le besoin d'étudier les biographies des grands traducteurs et traductologues, idée discutée aussi par Daniel Simeoni, ici même, dans sa dernière contribution, qui acquiert soudainement le statut de dernière volonté ou d'héritage post mortem. Il proposait en effet d'écrire une historiographie de la traductologie en insistant sur les parcours exiliques de nombreux traductologues du $\mathrm{XX}^{\mathrm{e}}$ siècle aux prises avec le processus complexe d'adaptation existentielle et intellectuelle dans un nouvel environnement.

Cela nous mène au deuxième point mentionné au début du paragraphe précédent, soit le changement de la perception de la traduction. Comme le postule Basalamah, la traduction est plus qu'une communication : elle est avant tout transmission. A l'heure actuelle, surtout à cause d'Internet qui est un moyen de production et de reproduction extrêmement efficace, les 
traducteurs se voient responsabilisés éthiquement à participer à la mise en place des conditions qui seraient favorables à une libre circulation des savoirs là où l'accès y est limité. Si la transmission s'inscrit normalement dans la continuité spatiotemporelle qui justifie pleinement l'investissement collectif (de génération en génération dans un contour social bien délimité), sinon entre égaux (le répertoire de la littérature mondiale en serait le summum), la transmission dans une dynamique actuelle présuppose le partage, la solidarité, la générosité et exige donc une posture clairement éthique. Puisque le contexte n'est plus un seul pays, une seule société, mais le monde, devenu virtuel grâce à Internet, la question est posée autrement par Basalamah qui réfléchit sur les possibilités éventuelles offertes par un " mouvement de résistance qui [..] remet en question les normes traditionnelles de l'auctorialité en encourageant le partage et l'accès libre aux "créations", [et qui] propose une alternative au pouvoir monopolistique des entités industrielles et commerciales mondialisées en revendiquant pour les usagers le droit de créer selon le principe transparent ("open source") [...], de la traduction " (p. 262). L'éducation à la traduction avancée par Cronin prend tout son sens dans cet enjeu important.

Dans «La traduction et ses discours ", Berman situe la théorie descriptive de Even-Zohar et de Toury dans les discours objectifs généraux qui sont produits généralement par les spécialistes sans avoir recours à la pratique de la traduction. Il considère également que cette théorie proposant une " science du traduit " doit être soumise à une critique systématique, justement parce qu'elle se veut descriptive, neutre et ancrée dans les phénomènes observables. Ainsi, la critique peut être accomplie soit en fonction de la société (normes) soit en fonction du sujet lui-même (éthique). Mais cette tension entre le social et l'individuel est déjà introduite par Toury lui-même dans Descriptive Translation Studies and Beyond (1995), quand il discute des deux stratégies régulatrices du comportement traductionnel : la standardisation et l'interférence dont le degré, comme le remarque Anthony Pym, ne dépend pas véritablement des facteurs socioculturels, mais plutôt du risque que le traducteur prend ou non (Pym, p. 325). 
En privilégiant l'observation pour comprendre en profondeur le phénomène étudié et pour en dégager les relations et les lois, le tournant descriptiviste a non seulement généré de nombreux projets de recherche dans les contextes et les problématiques extrêmement diversifiés, mais a aussi alimenté les débats épistémologiques et méthodologiques au sein de la discipline.

Il reste désormais à changer de centration, c'est-àdire à réfléchir sur l'herméneutique du sujet traduisant, ce qui permettrait d'ouvrir la porte à la dimension critique et éthique de la traduction, ainsi qu'aux circuits de traduction qui se forment $\mathrm{au}$-delà des frontières étatiques et qui rendent de plus en plus problématique ce concept crucial chez Toury : la norme.

\section{Aurelia Klimkiewicz Université York, Collège Glendon}

Luise von Flotow and Reingard M. Nischik, eds. Translating Canada. Charting the Institutions and Influences of Cultural Transfer: Canadian Writing in German/y. Ottawa, University of Ottawa Press, "Perspectives on Translation," 2007, 344 p.

This welcome contribution to Canadian Translation Studies has brought together fourteen essays, all written in English, that reflect on Canadian literature in German translation. Canadian literature is not to be understood here as exclusively English-Canadian, which is an all too common international misconception. To some extent the tendency to separate more or less categorically (English-)Canadian literature from Quebec literature stems from the labours of two autonomous and strong associations: the International Council for Canadian Studies and the Association internationale des études québécoises. Rather this collection has moved beyond the binary opposition that divides English-Canadian and Québécois literatures, for it includes essays on Franco-Quebec literature, minority Franco-Canadian literatures and First Nations' literature, as well as the expected Anglo-Canadian literature. Furthermore, a broad spectrum of genres are covered, for chapters have been devoted to the study 